\title{
Conversion from Calcineurin Inhibitors to Sirolimus Maintenance Therapy in Renal Allograft Recipients with Risk Factors
}

\author{
Shuming Ji ${ }^{1}$, Jiqiu Wen ${ }^{1}$, Dongrui Cheng ${ }^{1}$, Qiquan Sun ${ }^{1}$, Jinsong Chen ${ }^{1}$, Zhihong Liu ${ }^{2 *}$ \\ ${ }^{1}$ Research Institute of Nephrology, Department of Nephropathy, Jinling Hospital, Nanjing, China \\ ${ }^{2}$ Nanjing University School of Medicine, Nanjing, China \\ E-mail: *jishuming@medmail.com.cn
}

Received July 31, 2011; revised August 17, 2011; accepted August 23, 2011

\begin{abstract}
Background: The efficacy and safety of conversion treatment with sirolimus in renal transplant recipients using the calcineurin inhibitor (CNI) with one or more risk factors was evaluated. Methods: Ninety-three renal transplant recipients were prospectively enrolled. CNIs(CsA and FK506) as main immunosuppressant were converted to SRL immunosuppressant protocol. Rapid conversion with sirolimus was performed in all patients. The CNI withdrawal was in 2 weeks. At 4 hours after oral administration of cyclosporin A or tacrolimus, the patients took sirolimus. Initial dose of sirolimus was $6 \mathrm{mg}$, and repeated maintenance dose is 1.0 $2.0 \mathrm{mg} / \mathrm{d}$. The first concentration of sirolimus was detected at $5-7$ days after first oral administration, and the target concentration was $6-10 \mu \mathrm{g} / \mathrm{L}$. Results: The symptoms were markedly improved in patients with CNI induced renal toxicity and CNI induced liver toxicity, and the concentration of sirolimus were maintained at $(5.1 \pm 1.2) \mu \mathrm{g} / \mathrm{L}$. Serum creatinine levels decreased from $(297.72 \pm 150.28) \mu \mathrm{mol} / \mathrm{L}$ to $(123.76 \pm$ 44.2) $\mu \mathrm{mol} / \mathrm{L}$, and the liver function were recovery in $24(92.3 \%)$ patients. 9 patients with high glucose returned to normal, and 2 patients were improved. Serum creatinine levels decreased more than $25 \%$ of primary level in 17 patients, and the effective rate was 51.5\%. 10 patients with tumor were appeared $6-43$ months after renal transplantation, no recurrence was found in 8 of them and 2 patients were dead. Acute rejections were occurred in 3 patients at 6 months after conversion treatment. The complications were included hyperlipidemia and proteinuria. 3 patients were dead, 6 patients returned to dialysis treatment, and 2 patients were removal of grafts. At 3 years after conversion treatment, the survival rates of patients and grafts were $90.9 \%$ and $75.8 \%$, respectively. Conclusion: The conversion treatment with SRL and MMF may be a better option for the renal transplant recipients using the CNI with risk factors appeared.
\end{abstract}

Keywords: Renal Transplant, Calcineurin, Inhibitor, Conversion, Sirolimus

\section{Introduction}

Over the past two decades, the progress in renal transplantations has been focused much on the ways of reducing acute rejection. Incidence of acute rejections in the majority of renal transplant recipients has fallen below $20 \%$ and 1-year graft survival rate was as high as $90 \%$. This is resulted mainly from the use of calcineurin inhibitor (CNI), which could effectively prevent acute renal allograft rejection. However, the long-term survival of renal transplant recipients has not shown any improvement. [1] CNI-induced-nephrotoxicity, CNI-asso- ciated hepatotoxicity, post-transplant diabetes mellitus (PTDM), chronic allograft nephropathy(CAN), malignancy incidence rate as well as other adverse reactions resulted from CNI in renal transplant recipients, are the major risk factors. [2] In recent years, substituting the use of CNI with sirolimus (SRL) in part of the immunosuppression scheme for renal transplant recipients have been increasingly focused upon. [3] We had applied the newly revised immunosuppressive scheme, where SRL conversion was used instead of CNI when one or more risk factors had been identified to evaluate the efficacy and safety of conversion calcineurin inhibitor to siro- 
limus in the renal transplant recipients.

\section{Methods}

\subsection{Patients}

Ninety-three renal transplant recipients who had received conversion-to-SRL-based immunosuppressive therapy were prospectively enrolled at Jinling Hospital from June 2002 to December 2005. Patient demographics data are listed in Table 1. Criteria for inclusion were: I. Patients with CNI-induced nephrotoxicity is characterized by irreversible tubulointerstitial fibrosis in a striped pattern beginning in the medulla and progressing to the medullary rays of the cortex, generally accompanied by some degree of renal dysfunction; [4] II. Patients with CNI-associated-hepatotoxicity: [5] Bilirubin and transaminases increased significantly, but,bilirubin and transaminases decreased significantly within 2 weeks after withdrawal CNIs, and liver function normalized; III. Post-transplant diabetes mellitus was defined as the uninterrupted need for glucose-lowering medication for at least 3 months. [6] IV. Chronic allograft nephropathy characterized by progressive renal dysfunction accompanied by chronic interstitial fibrosis, tubular atrophy, vascular occlusive changes, and glomerulosclerosis. [7] V. Patients with concurrent post-transplant malignancy. Any patients of the following had not been included in this study: Those who had resumed back to their conventional immunosuppressive therapy due to disease progression or financial reasons; Those who had already hyperlipidemia (blood cholesterol $>6 \mathrm{mmol} / \mathrm{L}$, or triacylglycerol $>2 \mathrm{mmol} / \mathrm{L}$ ); urine protein $>0.8 \mathrm{~g} / 24 \mathrm{~h}$; peripheral blood $\mathrm{WBC}<3.5 \times 10^{9} / \mathrm{L}$, platelet count $<80 \times 10^{9} / \mathrm{L}$. All the clinical data collected in the paper for research is strictly complied with the Regulation of Human Organ
Transplantation in China, and no prisoners or organs from prisoners were used in the collection of data for this study.

\subsection{Switch Scheme from CNI to Sirolimus}

SRL conversion course, applies fast conversion, [8] the two drugs are overlapped for a shorter period, between one and two weeks, generally reducing CNI by $50 \%$ starting from the day that SRL is introduced. The objective of this approach would be to maintaining CNI until being sure that SRL levels are sufficient. Initial single oral loading dose was given at $6 \mathrm{mg}$, followed by maintenance dose given $1-2 \mathrm{mg} /$ day. SRL target trough levels were 6 - $10 \mathrm{ug} / \mathrm{L}$ (high-performance liquid chromatography). The first level is usually measured between 5 and 7 days after initiating SRL, and if it is within or close to the target range, CNI is then discontinued. If the level is still low, the SRL dose is increased and CNI is maintained until measuring a second level a week later. After conversion, mycophenolate mofetil (MMF,750 mg, twice per day) adjusted by AUC 0-12, and maintained at 35 $45 \mathrm{mg} \cdot \mathrm{h} / \mathrm{L}$. Steroid therapy was unchanged. The study was approved by the committee of ethics at Jinling Hospital. All patients gave their written informed consent.

\subsection{Clinical Design}

Serum creatinine levels, rate of acute rejection, renal graft loss, pulmonary infection and mortality rate were monitored dynamically after SRL conversion had been initiated. Those specific criteria for observations include liver and renal function, blood cholesterol, triacylglycerol, blood sugar, urine protein and routine peripheral blood tests. In the meantime, SRL blood trough concentrations were monitored.

Table 1. Clinical parameters at SRL conversion.

\begin{tabular}{|c|c|c|c|c|c|}
\hline & CNI-nephrotoxicity & CNI-hepatotoxicity & PTDM & CAN & Tumor \\
\hline $\mathrm{n}$ & 13 & 26 & 11 & 33 & 10 \\
\hline Age (year) & $38.2 \pm 11.1$ & $36.7 \pm 14.1$ & $40.2 \pm 8.0$ & $34.2 \pm 9.9$ & $41.0 \pm 6.4$ \\
\hline \multicolumn{6}{|c|}{ Basal immunosuppressive drugs at conversion (n) } \\
\hline CsA & 10 & 21 & 2 & 32 & 7 \\
\hline FK506 & 3 & 5 & 9 & 1 & 3 \\
\hline MMF & 13 & 26 & 11 & 33 & 9 \\
\hline Transplantation time (mons) & $22 \pm 6$ & $23 \pm 4$ & $25 \pm 9$ & $30 \pm 9$ & $36 \pm 3$ \\
\hline
\end{tabular}

CNI: calcineurin inhibitor, PTDM: Post-transplantation diabetes mellitus, CAN: Chronic allograft nephropathy. 


\subsection{Statistical Analysis}

Values before and after conversion were compared with a Wilcoxon signed rank test. SPSS (version 11) software was used to make the calculations. Values of $\mathrm{P}<0.05$ were considered to be significant.

\section{Results}

\subsection{Demographic Characteristics}

Patient demographics data are listed in Table 1. CNIinduced nephrotoxicity $(13,13.9 \%)$, CNI-associated heaptotoxicity $(26,28.0 \%), \operatorname{PTMD}(11,11.8 \%), \mathrm{CAN}(33,35.5 \%)$ and post-transplantation tumor $(10,10.8 \%)$. Follow-up arrangements were conducted at $36.9 \pm 1.2(35-38)$ months post-SRL therapy, mean SRL dose was $2.6 \pm 0.4$ $\mathrm{mg} / \mathrm{d}, \mathrm{SRL}$ target trough levels were $7.3 \pm 2.3 \mathrm{ug} / \mathrm{L}$.

\subsection{Clinical Outcomes after SRL Conversion}

CNI-induced nephrotoxicity was reported in 13 patients (10 from CsA and 3 from FK506), average CNI maintenance time given before conversion was $11 \pm 6$ months and average blood trough levels were $213.9 \pm 19.3 \mathrm{ug} / \mathrm{L}$ and $10.3 \pm 3.7 \mathrm{ug} / \mathrm{L}$ respectively. Meanwhile other clinical complications such as hirsutism, gingival hypertrophy and elevated blood pressure were noted. After SRL conversion, the above symptoms as mentioned showed significant improvement, where blood SRL concentration was maintained at $7.1 \pm 1.2 \mathrm{ug} / \mathrm{L}$, and serum creatinine level was reduced from $3.3 \pm 1.7 \mathrm{mg} / \mathrm{dl}$ to $1.4 \pm$ $0.5 \mathrm{mg} / \mathrm{dl}$.

CNI-associated hepatotoxicity was reported in 26 patients (21 from CsA and 5 from FK506). Serum alanine aminotransferase (ALT) was 102.2 $\pm 24.3 \mathrm{U} / \mathrm{L}$, total bilirubin (TB) was $38.2 \pm 9.8$ umol/L.After conversion, ALT was $30.1 \pm 13.3 \mathrm{U} / \mathrm{L}$, TB was $11.8 \pm 7.2 \mathrm{umol} / \mathrm{L}$, and the period of liver functioning restoration after conversion took $11.3 \pm 1.9$ days; ALT was noted reduced but not reaching its normal level in two cases, in which concurrent hepatitis $\mathrm{C}$ was noted in one patient whose blood direct bilirubin levels showed progressive elevation, and succumbed to liver failure 6 months after SRL conversion.

Hyperglycemia had been reported in 11 patients (2 from CsA and 9 from FK506) where blood glucose levels have returned to normal in 9 cases within 6 months after conversion. Oral hypoglycemic drugs had been stopped and glycosylated hemoglobin returned to normal from $9.2 \pm 0.6$ to $5.4 \pm 0.3 \%$. The other two patients had switched from insulin to oral hypoglycemics in control of blood glucose levels efficiently.
CAN was confirmed on allograft pathology $38 \pm 3$ months after SRL conversion. In 17 cases, fall of serum creatinine exceeded $25 \%$ compared with their original level with an efficacy rate of 51.5\%. Among the other 16 cases which were $\mathrm{SCr}>2.5 \mathrm{mg} / \mathrm{dl}$ before SRL conversion, improvement in renal functioning was achieved in 2 cases, whereas no improvement shows in the other 14 cases. Serum creatinine levels in the 14 patients showed slow-progressive rise, in which performing dialysis was necessary in 5 patients, and allograft removal was managed in one patient.

Tumor occurrences were noted around 6 - 43 months. Among them, bladder cancer was reported in 3 cases, breast cancer in 3 cases, colon cancer, esophageal cancer, thyroid cancer and lymphoma in 1 case each. In the 3 patients with bladder transitional cell carcinoma (T2, grade-II), transurethral resection of tumor was managed followed by regular bladder instillation chemotherapy. Patients were followed-up for 36 - 38 months, no recurrences were reported in 2 cases. However, recurrence was reported in one case after 11 months. A second operation of transurethral resection of tumor had been arranged and no further recurrence has been detected till today.; Among the 3 patients with breast cancer, 2 patients presented with grade III invasive ductal carcinoma of the right breast, where cancerous growth was detected near the base region plus ipsilateral axillary lymph node metastasis (immunohistochemistry: E-cad+++, EMC +++ ). Surgical resection followed by standard chemotherapy had been managed and no recurrence was reported after 37 months of follow-up. One patient with colon cancer had been managed with chemotherapy alone, however, the patient succumbed to massive gastrointestinal tract hemorrhage 8 months after SRL conversion. Esophageal cancer and thyroid cancer, 1 patient of each had received surgical resection of tumor only, and no recurrence was reported after 37 months and 38 months follow-up respectively; One patient who had been diagnosed with lymphoma was treated with intermittent chemotherapy. The patient survived for 36 months and succumbed to pulmonary infection subsequently.

\subsection{Adverse Reactions}

Acute rejection occurred within 6 months after SRL conversion in 3 cases $(9.1 \%)$, which were in the third month in 1 case and the fourth month in the other 2 respectively. Methylprednisolone pulse therapy was managed and blood SRL and MMF concentrations were readjusted. One month later, total recovery from acute rejection was achieved in one patient, SCr level had returned to normal $(1.1 \mathrm{mg} / \mathrm{dl})$. The other 2 cases were beyond control and had to be managed with hemodialy- 
sis, in which allograft removal was managed in one patient. Pulmonary infections were reported in 3 cases 6 months after SRL conversion (9.1\%), which were from the PTDM, CAN and tumor conversion groups. Among them, one patient with lymphoma succumbed to infection (mixed pulmonary infection: cytomegalovirus plus staphylococcus and fungal agents), whereas recovery was achieved in the other 2. Mild nausea and vomiting and severe diarrhea were 9 cases $(27.3 \%)$, and they were all alleviated when drug doses had been reduced. Hypercholesterolemia was 11 cases $(33.3 \%)$, hypertriacyglycerolemia in 12 cases $(36.4 \%)$, hyperuricemia in 9 cases $(27.3 \%)$, liver dysfunction in 8 cases $(24.2 \%)$, leukopenia in 8 cases $(24.2 \%)$, thrombocytopenia in 6 cases $(18.2 \%)$ and oral ulcers in 5 cases $(15.2 \%)$. After 36 months of follow-up, proteinuria $(3.2 \pm 0.5 \mathrm{~g} / 24 \mathrm{~h}$.) was reported in 13 cases (39.4\%). Tripterygium Wilfordii Hook f. $(60 \mathrm{mg} / \mathrm{d})$ was concomitantly added into treatment, [9] and full recovery was achieved in 9 cases $(69.2 \%)$.

\subsection{Survival Rates}

Within 3 years of follow-up, death was reported in 3 cases, where their causes of death include liver failure, severe pulmonary infection and massive gastrointestinal tract hemorrhage one of each case. Serum creatinine in 8 patients showed progressive elevation (CAN 6 cases, acute rejection 2 cases), in which 6 of them had to resume dialysis and 2 required allograft removal. The 3-year kidney graft and patient survival rates with SRL conversion were $75.8 \%$ and $90.9 \%$ respectively.

\section{Discussion}

Sirolimus, as a new generation, highly-effective immunosuppressive agent, has little nephrotoxicity along with anti-proliferative as well as anti-tumor effects. [10] Application of SRL has provided the opportunity for renal allograft recipients to withdraw from the use of $\mathrm{CNI}$, thus becoming the clinical research hotspot of how to prevent post-transplantation long-term complication, and improve the long-term survival of renal transplants. [11] The question on how to select the most appropriate individual for safe and effective conversion on renal allograft recipients from one immunosuppressive agent to another has brought aroused concerns from many patient.

\subsection{Suitability for Conversion to SRL Therapy}

In our study, the efficacy rate of conversion treatment in CAN patients was $51.5 \%$, but of course, the renal function on some patients did not show any improvement on schedule, or was even deteriorated. From our study, we have discovered that the mean initial serum creatinine level before conversion in patients with no renal function improvement after conversion to SRL, was high. Therefore, the therapeutic efficacy of conversion treatment is rising if applying to CAN patients with initial serum creatinine $<2.5 \mathrm{mg} / \mathrm{dl}$. Hence, early intervention is a leading factor towards successful conversion treatment. [12] So giving early conversion treatment before renal impairment develops should be considered. Our experience tells us that conversion treatment shouldn't be intervened when serum creatinine levels are high $(>2.5$ $\mathrm{mg} / \mathrm{dl}$ ), which means that we shouldn't wait until creatinine reaches to this level. [13]

In our case studies, restoration of hepatic function was reported in 24 patients after conversion treatment. In spite of the presence of hepatic dysfunction resulted from SRL as reported in our study, the mechanism may not be similar to CNI-induced hepatic function impairment. Besides, dose-related adverse reaction could be an essential factor, so as long as timely dose adjustment is assured by giving a rational dosage which corresponds to the desired target concentration of $6-10 \mathrm{ug} / \mathrm{L}$, hepatic damage can be reduced or even avoided. Therefore, for some renal allograft recipients who have developed hepatic dysfunction upon receiving CNI-based treatment, switching to SRL may be a good choice.

In patients who've developed impaired glucose tolerance or diabetes mellitus after transplantation are suitable for SRL conversion and withdraw from CNI at the same time (particularly, FK506). Regardless of gradual steroid withdrawal done before or after conversion, a series of data have shown good results which was obtained from our conversion treatment. In our study, 9 PTDM patients who'd received conversion treatment achieved better control, which could have been associated with the elimination or reduction of CNI damage to pancreatic islets. This prompts the use of SRL does not increase glucose tolerance impairment in renal recipients, which makes immunosuppressive conversion to be the best option for the treatment of certain hyperglycemic recipients.

The arise of malignant tumor during the stable phase of post-transplantation has become an extremely prominent problem, which affects graft and recipient's longterm survival rates. [14] The classical strategies of controlling post-transplant malignancy include reducing the dose of administrated immunosuppressive agent, or even withdrawal. However, this could also impact immune responses significantly, and as a result, increases the risk of allograft impairment/loss. Conversion to SRL therapy may, in one hand, prevent the increase of immune response risk which brings rise to rejection; and on the 
other hand, as shown from some conversion treatment results, it has a suppressive effect on primary tumors as well as on metastasis theoretically.

\subsection{Safety of SRL Conversion}

The main adverse reactions noted from SRL conversion were hyperlipidemia, proteinuria, leucopenia, anemia and pulmonary infection in severe cases. These manifestations occurred mostly with the standard dose administration and blood trough levels $(10-15 \mathrm{ug} / \mathrm{L})$ recommended by foreign experts, and as we know that the adverse reactions are associated with its doses and concentration given, by lowering SRL doses and concentration should be able to reduce or prevent from these adverse reactions to occur; therefore, we think that there could possibly be differences between pharmacokinetic and drug metabolism gene polymorphism as well as genetic background variation in immune system between the Western and Asian population, $[15,16]$ i.e. similar to the administration of cyclsporine, we must not adopt the C2 monitoring standards of European of American renal recipients. To determine the appropriate SRL plasmadose concentration at variable intervals after transplantation in the Chinese population of renal transplant recipients will be the clinical research issue that requires a solution from now on.

In conclusion, Ninety-three renal transplant recipients were prospectively enrolled. CNIs(CsA and FK506) as main immunosuppressant were converted to SRL immunosuppressant protocol.These observations support the conversion treatment with sirolimus in renal transplant recipients using the calcineurin inhibitor (CNI) with one or more risk factors was effective and safe. At 3 years after conversion treatment, the survival rates of patients and grafts were $90.9 \%$ and $75.8 \%$, respectively. The results from this clinical trial suggest the conversion treatment with SRL and MMF may be a better option for the renal transplant recipients using the CNI with risk factors appeared.

\section{REFERENCES}

[1] L. Li and Q. Sun, "Renal transplantation in China: Ten Years of Experience at Nanjing Jinling Hospital," Clinical Transplants, Vol. 1, No. 5, 2006, pp. 71-77.

[2] A. Magnasco, A. Rossi, P. Catarsi, R. Gusmano, F. Ginevri, F. Perfumo and G. M. Ghiggeri, "Cyclosporin and Organ Specific Toxicity: Clinical Aspects, Pharmacogenetics and Perspectives," Current Clinical Pharmacology, Vol. 3, No.3, 2008, pp. 166-173. doi: $10.2174 / 157488408785747674$

[3] D. W. Hanto and R. Chudzinski, "What Does the CONVERT Trial Really Tell Us about Conversion from Cal- cineurin Inhibitors to Sirolimus?" Transplantation, Vol. 87, No. 2 , 2009, pp. 164-165. doi:10.1097/TP.0b013e318192790f

[4] J. R. Chapman, "Chronic Calcineurin Inhibitor Nephrotoxicity-Lest We Forget," American Journal of Transplantation, Vol. 11, No. 4, 2011, pp. 693-697. doi:10.1111/j.1600-6143.2011.03504.x

[5] R. Pfitzmann, J. Klupp, J. M. Langrehr, M. Uhl, R. Neuhaus, U. Settmacher, T. Steinmüller and P. Neuhaus, "Mycophenolatemofetil for Immunosuppression after Liver Transplantation: A Follow-up Study of 191 Patients," Transplantation, Vol. 76, No. 1, 2003, pp. 130-136. doi:10.1097/01.TP.0000071522.74885.48

[6] D. R. Kuypers, K. Claes, B. Bammens, P. Evenepoel and Y. Vanrenterghem," Early Clinical Assessment of Glucose Metabolism in Renal Allograft Recipients: Diagnosis and Prediction of Post-Transplant Diabetes Mellitus (PTDM)," Nephrology Dialysis Transplantation, Vol. 23, No. 6, 2008, pp. 2033-2042. doi:10.1093/ndt/gfm875

[7] K. Solez, R. B. Colvin, L. C. Racusen, B. Sis, P. F. Halloran, P. E. Birk, P. M. Campbell, M. Cascalho, A. B. Collins, A. J. Demetris, C. B. Drachenberg and I. W. Gibson, "Banff 05 Meeting Report: Differential Diagnosis of Chronic Allograft Injury and Elimination of Chronic Allograft Nephropathy ('CAN')," American Journal of Transplantation, Vol. 7, No. 3, 2007, pp. 518-526. doi:10.1111/j.1600-6143.2006.01688.x

[8] J. C. Ruiz, A. Alonso, M. Arias, J. M. Campistol, M. G. Molina, J. M. G. Posada, J. M. Grinyo, J. M. Morales, F. Oppenheimer, A. Sánchez Fructuoso and J. Sán-chezPlumed, "Conversion to Sirolimus," Nefrologia, Vol. 26, No. 2, 2006, pp. 52-63.

[9] S. M. Ji, L. S. Li, J. Q. Wen, G. Z. Sha, Z. Cheng, D. R. Cheng, J. S. Chen and Z. H. Liu, "Therapeutic effect of Tripterygium Wilfordii Hook f. on Proteinuria Associated with Sirolimus in Renal Transplant Recipients," Transplant Proceedings, Vol. 40, No. 10, 2008, pp. 3474-3478. doi:10.1016/i.transproceed.2008.07.140

[10] W. Ji-qiu and J. Shu-ming, "Alleviation of Calcineuron Inhibitor-induced Nephrotoxicity with Rapamycin," Chinese Journal of Nephrology Dialysis \& Transplantation, Vol. 16,No. 3, 2007, pp. 283-288

[11] J. Shu-ming, W. Ji-qiu, S. Guo-zhu, C. Dong-rui, S. Qi-quan, C. Jin-Song, L. Zhi-hong and L. Lei-shi, "Conversion Treatment with Sirolimus in Renal Transplant Recipients," Journal of Clinical Rehabilitative Tissue Engineering Research, Vol. 13, No. 31, 2009, pp. 60196022.

[12] A. Gürkan, S. Kaçar, U Erdoğdu, C Varilsüha, G. Kandemir, C. Karaca and F. Akman, "The Effect of Sirolimus in the Development of Chronic Allograft Nephropathy," Transplant Proceedings, Vol. 40, No. 1, 2008, pp. 114-116. doi:10.1016/j.transproceed.2007.11.005

[13] S. M. Ji, L. S. Li, G. Z. Sha, J. S. Chen and Z. H. Liu, "Conversion from Cyclosporine to Tacrolimus for Chronic Allograft Nephropathy," Transplant Proceedings, Vol. 39, No. 5, 2007, pp. 1402-1405. doi:10.1016/j.transproceed.2006.11.030

[14] J. M. Campistol, J. Albanell, W. Arns, I Boletis, J. Dantal, 
J. W. de Fijter, S. A. Mortensen and H. H. Neumayer, "Use of Proliferation Signal Inhibitors in the Management of Post-Transplant Malignancies-Clinical Guidance," Nephrology Dialysis Transplantation, Vol. 22, No. S1, 2007, pp. i36-i41. doi:10.1093/ndt/gfm090

[15] M. Schubert, RVenkataramanan, D. W. Holt, L. M. Shaw, W. McGhee, J. Reyes, S. Webber and R. Sindhi, "Pharmacokinetics of Sirolimus and Tacrolimus in Pediatric Transplant Patients," American Journal of Transplanta- tion, Vol. 4, No. 5, 2004, pp. 767-773. doi:10.1111/j.1600-6143.2004.00411.x

[16] X. Y. Qiu, Z. Jiao, M. Zhang, L. J. Zhong, H. Q. Liang, C. L. Ma, L. Zhang and M. K. Zhong, "Association of MDR1, CYP3A4*18B, and CYP3A5*3 Polymorphisms with Cyclosporine Pharmacokinetics in Chinese Renal Transplant Recipients," European Journal of Clinical Pharmacology, Vol. 64, No. 11, 2008, pp. 1069-1084. doi: $10.1007 / \mathrm{s} 00228-008-0520-8$ 